\title{
ESTÁNDARES PARA E-LEARNING ADAPTATIVO Y ACCESIBLE
}

\author{
(STANDARDS FOR ADAPTIVE AND ACCESSIBLE E-LEARNING)
}

\author{
Ramón Fabregat Gesa \\ Germán Darío Moreno García \\ Universitat de Girona (España)
}

Fernando Alonso Amo

José Luis Fuertes Castro.

Ángel Lucas González Martínez

Loïc Martínez Normand

Universidad Politécnica de Madrid (España)

\section{RESUMEN}

En la actualidad existe un gran número de estándares que tratan sobre la accesibilidad de los sistemas de e-Learning, desde diversos puntos de vista, como los modelos de usuario, los escenarios de aprendizaje, las preferencias de interacción, las capacidades de los dispositivos y metadatos para especificar cómo entregar cualquier recurso de acuerdo a las necesidades de los usuarios. También existen otros estándares relevantes en el campo de la accesibilidad y la usabilidad del software. Cada uno de estos estándares representa un punto de vista diferente con su propio conjunto de objetivos y alcance, lo que dificulta comprender las relaciones que existen entre todos ellos. Este artículo presentará una recopilación actualizada de los principales estándares relacionados con la accesibilidad, usabilidad y adaptabilidad de los sistemas de e-Learning en el contexto del proyecto de investigación A2UN@, que está centrado en atender las necesidades de accesibilidad y adaptación de todos, en la Educación Superior.

Palabras clave: adaptación, accesibilidad, e-Learning, estándares.

\begin{abstract}
Currently there are many standards that deal with accessibility issues regarding users' models, learning scenarios, interaction preferences, devices capabilities, metadata for specifying the delivery of any resource to meet users' needs, and software accessibility and usability. It is difficult to understand the existing relationships between these standards, as each one represents a different viewpoint and thus has its own sets of goals and scope. This paper gives an overview on existing standards addressing
\end{abstract}


accessibility, usability and adaptation issues in e-learning, and discusses their application to cope with the objectives of the A2UN@ project, which focuses on attending the accessibility and adaptation needs for ALL in Higher Education.

Keywords: adaptation, accessibility, e-Learning, standards.

Los sistemas de formación deberían ofrecer un servicio inclusivo e individualizado al considerar las necesidades específicas de cada alumno. Para ello dichos sistemas deberían adaptar sus procesos formativos en función del contexto, del entorno, de los dispositivos que utiliza cada usuario, así como en función de las competencias, destrezas y habilidades individuales.

Esto es especialmente importante en los sistemas de e-Learning, basados en el uso de las Tecnologías de la Información y la Comunicación (TIC), y que están dirigidos a ofrecer su servicio de forma remota, con lo que se amplían las posibilidades de cobertura de toda la sociedad. Las TIC desempeñan hoy en día un papel cada vez más importante en el apoyo del proceso de e-Learning de estudiantes y profesionales con necesidades específicas, incluidas las personas con diversidad funcional, o personas con discapacidad. Y todo esto cobra más importancia cada día, con el auge de paradigmas tales como el "Diseño para todos", el "Acceso para todos" y la formación durante toda la vida o aprendizaje permanente (LLL, del inglés life-long learning). Puede definirse LLL como la formación durante toda una vida, voluntaria y por motivación propia, debida a razones personales o profesionales. La accesibilidad es especialmente relevante en este paradigma LLL, debido a la evolución de las capacidades humanas al envejecer.

Actualmente las TIC utilizadas en el contexto del e-Learning no se pueden considerar aun como plenamente inclusivas, a pesar de los grandes avances realizados en el campo de la accesibilidad electrónica en los últimos años. Por esta razón, tanto las iniciativas europeas como las legislaciones nacionales, promueven y regulan acciones y estándares que permiten generar las condiciones para que todos los ciudadanos puedan participar en la sociedad de la información proporcionando los servicios, procedimientos e información de manera accesible para cada persona. También se definen políticas para crear una sociedad que está dispuesta a los cambios tecnológicos en el momento en que ocurren.

Este artículo se plantea en el contexto del proyecto de investigación A2UN@, su objetivo es analizar la posibilidad de desarrollar un marco de trabajo TIC, basado 
en estándares y modelado de usuario, para dar soporte al desarrollo de los servicios LLL, requeridos para atender las necesidades de accesibilidad y adaptación de todos en la Educación Superior.

Este artículo recoge los resultados del análisis de estándares relacionados con la accesibilidad, usabilidad y adaptación de e-Learning en el contexto del proyecto A2UN@. La siguiente sección introduce brevemente los objetivos y alcance del proyecto, haciendo especial hincapié en su estrategia de utilización de estándares. El apartado 3 proporciona una enumeración y análisis de aquellos estándares que se han considerado más relevantes para el proyecto. Finalmente trata, a modo de conclusiones, sobre la posibilidad de crear un modelo genérico para un sistema LLL basado en estándares.

\section{EL PROYECTO A2UN@: ACCESIBILIDAD Y ADAPTACIÓN PARA TODOS EN LA EDUCACIÓN SUPERIOR}

El proyecto A2UN@ “Accesibilidad y adaptación para todos en la Educación Superior”, está financiado por el Ministerio Español de Ciencia e Innovación. Comenzó en enero de 2009 y tendrá una duración de 3 años. El proyecto es responsabilidad de la Universidad Nacional de Educación a Distancia (UNED) y la Universidad de Girona (UdG), con la colaboración de investigadores de la Universidad Politécnica de Madrid (UPM). La UNED coordina el proyecto.

El objetivo principal del proyecto es crear un marco general de las TICS para apoyar el desarrollo de los servicios de LLL requeridos para atender las necesidades de adaptación y accesibilidad para todos en la Educación Superior (ES). Para este fin el proyecto se ha estructurado mediante una serie de paquetes de trabajo, incluyendo las siguientes áreas: (1) Estándares que soportan la accesibilidad de los objetos y servicios de aprendizaje, (2) Modelado del usuario y soporte dinámico, (3) Flujos de trabajo y servicios de aprendizaje adaptables y reutilizables, y (4) Modelado de dispositivos, interfaces de usuario adaptativas y estrategias de negociación. En otros términos, el objetivo de este proyecto también podría ser planteado cómo detectar, extender, interrelacionar, integrar y aprovechar a la medida de lo posible todos los ámbitos en el que pueda definirse un marco general, flexible, abierto, y basado en estándares para soportar el desarrollo del paradigma del aprendizaje permanente.

Por otra parte, se puede evidenciar la existencia de una necesidad dirigida a lograr la interoperabilidad en los diferentes niveles de abstracción de las tecnologías de aprendizaje teniendo en cuenta la gran variedad de servicios, contenidos y 
dispositivos que existen. Esta necesidad plantea como desafío el desarrollo de la infraestructura necesaria para facilitar la definición, desarrollo, implantación y evaluación de los servicios que se proporcionan para apoyar el aprendizaje de una manera accesible y personalizada en la Educación Superior.

Con el propósito de desarrollar estos objetivos, el proyecto A2UN@ sigue una estrategia de uso activo de diferentes estándares. La pregunta que se planteó al iniciar el proyecto es: ċla implementación de estándares aceptados mundialmente, dentro de un entorno global y dinámico, puede hacer frente a desafíos de la sociedad moderna tal como la aplicación del aprendizaje permanente? Nuestra hipótesis inicial es positiva y se basa en dos premisas:

- Uso generalizado: para asegurar el éxito de un producto, servicio o tecnología, ésta debe ser aceptada, implementada y utilizada por una gran mayoría de personas. Desde el proyecto se piensa que el uso de estándares internacionales es un medio adecuado para recopilar los conocimientos sobre un tema determinado con un grado suficiente de representación de las partes interesadas.

- Punto de partida: al desarrollar el proyecto no se pretende reinventar la rueda. El uso de estándares es un buen punto de partida para comprobar y analizar el conocimiento adquirido hasta la fecha sobre un tema específico y, desde allí, tratar de ampliarlo tanto como nos sea posible.

Por otra parte, los participantes en el proyecto consideran que el conocimiento sobre un tema es dinámico y debe ser adecuado a las necesidades dela humanidad. Por lo tanto, otro objetivo del proyecto A2UN@ es trabajar activamente en la extensión y la evolución de los estándares para reflejar los nuevos resultados (Martínez, 2007).

\section{ESTÁNDARES RELEVANTES PARA A2UN@}

Aquí se presentan los resultados del análisis de estándares que pueden servir de base para el desarrollo del proyecto A2UN@. Se ha estructurado esta descripción en dos apartados. En el primero se presentan los criterios utilizados para elegir estándares relevantes y se enumeran y describen brevemente dichos estándares (se han recopilado 43 documentos). En el segundo se presenta un análisis de estos estándares de acuerdo con los pilares del proyecto A2UN@. 
Se han utilizado como fuentes clave de información el informe sobre estándares relacionados con accesibilidad preparado por Richard Hodgkinson para el Instituto Real Británico de Personas Ciegas (en inglés Royal National Institute for the Blind) (Hodgkinson, 2009) y el inventario de estándares publicado en el informe técnico internacional ISO/IEC TR 29138-2 (ISO/IEC, 2009).

\section{Visión general}

Para seleccionar los estándares que fueran relevantes en el ámbito de la accesibilidad y adaptación para todos en sistemas de e-Learning, se han tenido en cuenta los siguientes criterios:

- Los estándares están relacionados con las áreas de investigación de nuestro proyecto.

- Se trata de pautas publicadas por consorcios internacionales o bien estándares publicados por organismos oficiales de normalización internacionales.

- Son documentos que prestan especial atención a la accesibilidad o a la usabilidad.

Los documentos que se han seleccionado han sido producidos por las siguientes entidades:

- $\quad \mathrm{ADL}$ (Advanced Distributed Learning Initiative) una iniciativa que tiene su origen en la oficina de la Secretaría de Defensa de Estados Unidos, y que trabaja en la introducción se sistemas de enseñanza distribuidos en el entrenamiento del personal de defensa.

- CETIS (Centre for Educational Technology and Interoperability Standards) es un Centro del Reino Unido, encargado de dar soporte sobre tecnologías y estándares educativos al sector de la educación superior.

- $\quad$ ETSI (European Telecommunications Standards Institute) es uno de los tres organismos oficiales de normalización europeos. ETSI produce estándares para las TIC, incluyendo tecnologías fijas, móviles, de radio, convergentes, de radiodifusión y de Internet. 
- IEEE (Institute of Electrical and Electronics Engineers) esta asociación de ingenieros de ámbito global incluye, entre sus acciones, el desarrollo de estándares sobre diversos aspectos de la ingeniería eléctrica y electrónica, incluyendo la computación y el software.

- IMS GLC (IMS Global Learning Consortium) es un consorcio internacional en el que participan proveedores de tecnologías educativas, proveedores de contenidos, instituciones educativas y organizaciones gubernamentales. $\mathrm{Su}$ objetivo es mejorar la educación y el aprendizaje mediante la aplicación estratégica de la tecnología.

- ISO (International Standards Organization) es un organismo oficial de normalización de ámbito internacional. Desarrolla estándares e informes técnicos en una gran cantidad de ámbitos, incluyendo algunos relacionados con las TIC.

- IEC (International Electrotechnical Commission) es otro organismo oficial de normalización de ámbito internacional, aunque más centrado en electricidad y electrónica de consumo. ISO e IEC colaboran en el desarrollo de normas conjuntas en el ámbito de la computación, a través de un comité técnico conjunto.

- $\quad \mathrm{W}_{3} \mathrm{C}$ (World Wide Web Consortium) es un consorcio internacional formado por empresas, universidades, organizaciones de usurarios, etc., cuya misión es liderar el desarrollo de las tecnologías que se usan en la web.

A continuación se presentan brevemente los documentos que se han analizado, ordenados alfabéticamente según su código completo. Se ha mantenido su título en inglés para facilitar su referencia posterior

\section{ADL SCORM - Learning object reference model}

SCORM (del inglés Sharable Content Object Reference Model) es una especificación de ADL. La última versión se denomina SCORM 2004, y ha sido revisada varias veces. Actualmente está publicada la cuarta edición (ADL, 2009).

Se trata de una colección de estándares y especificaciones para enseñanza virtual basada en tecnologías web. Define las comunicaciones entre el contenido en el lado del cliente y un sistema servidor llamado entorno de ejecución. SCORM también decide qué contenido se puede empaquetar en un fichero ZIP. 
Los tres documentos de referencia son SCORM CAM (del inglés Content Aggregation Model), SCORM RTE (de Run Time Environment) y SCORM SN (de Sequencing and Navigation).

\section{CETIS LEAP2A - Portability and interoperability of e-portfolio}

Esta especificación, titulada en inglés “The LEAP2A specification for portability and interoperability of e-portfolio information" (CETIS, 2009) trabaja sobre el concepto de portfolio electrónico ("e-portfolio"), donde una persona puede recopilar información relativa a su formación, logros y habilidades. Un estudiante usa un "e-portfolio" para recoger evidencias de su formación, de cara a la búsqueda de empleo.

La especificación LEAP2A ha sido desarrollada para dar soporte a la interoperabilidad entre las herramientas de "e-portfolio" y sistemas similares. Para desarrollar esta especificación se ha usado el formato Atom (Atom Syndication Format). Los elementos principales especificados con LEAP2A son: habilidad, logro, actividad, reunión, organización, persona, plan, recurso, selección.

\section{ETSI EG 202116 V1.2.1 - Design for all guidelines for ICT products and services}

Este documento (ETSI, 2002) proporciona pautas destinadas a los diseñadores de productos y servicios TIC sobre factores humanos, buenas prácticas de diseño y estándares relevantes nacionales e internacionales. Estas pautas pretenden promover el enfoque del diseño para todos de forma que se diseñen productos y servicios que sean accesibles para el mayor número de personas posibles, incluyendo personas con discapacidad y personas mayores, sin que sea necesario adaptarlos o hacer diseños específicos. Este documento se aplica a cualquier producto y servicio TIC con interfaz de usuario que pueda conectarse a redes de comunicaciones móviles o fijas.

\section{ETSI ES 202746 V1.1.1 - User profile preferences and information}

Este documento (ESTI, 2010) define un conjunto de parámetros de configuración de preferencias y de información de perfiles de usuario para que sean aplicados en servicios y dispositivos TIC, de forma que los puedan utilizar sus usuarios y proveedores. El documento especifica: 
- Objetos, incluyendo parámetros, valores, operaciones y un léxico de términos de usuarios finales.

- Un lenguaje de reglas para definir funcionalidades como la modificación automática de perfiles de usuario.

Este documento incluye en su objeto y campo de aplicación tres tipos de soluciones de perfiles de usuario:

- Aquellas soluciones destinadas al beneficio del usuario final.

- Aquellas soluciones en las cuales el usuario final tiene derechos para gestionar directamente los contenidos del perfil.

- Aquellas soluciones en las cuales el usuario tiene el derecho de negociar con la entidad que posee la información sobre los contenidos del perfil.

\section{IEEE std. 1484.1-2003 - Learning Technology Systems Architecture}

Este estándar de IEEE (IEEE, 2003) propone una arquitectura de alto nivel para sistemas de e-Learning, llamada LTSA (del inglés Learning Technology Systems Architechture). La arquitectura describe el diseño de estos sistemas y sus componentes principales. Trata de ser neutra en lo que respecta a métodos pedagógicos, contenidos, cultura, implementaciones y plataformas. Con ello proporciona un marco de trabajo para comprender sistemas existentes y futuro, y promueve la interoperabilidad y la portabilidad al proporcionar interfaces de sistema abstractas y de alto nivel.

IEEE std. 1484.4-2007 - Digital Rights Expression Languages for eLearning

Este documento (no es un estándar, sino un documento de nivel inferior: "Recommended Practice") facilita la creación, gestión y entrega de contenido digital para e-Learning que implementen los Lenguajes de Expresión de Derechos Digitales (DREL). Con este documento se determinan qué extensiones son necesarias en los DREL para cubrir los requisitos que se han identificado desde el punto de vista de sistemas de enseñanza (IEEE, 2007). 
IEEE std. 1484.11.1-2004 - Data model for content to learning system communication

Este estándar (IEEE, 2004) describe un modelo de datos para dar soporte al intercambio de elementos de datos y sus valores entre un objeto de contenido de aprendizaje (LOM, del inglés Learning Object Metadata, basado en el estándar 1484.12.1) y un sistema de gestión de aprendizaje (LMS, del inglés Learning Management System). Este estándar está basado en un modelo de datos del mundo aeronáutico definido en el documento "Computer Managed Instruction Guidelines For Interoperability”, modelo que ha sido adaptado al caso de sistemas de aprendizaje.

IEEE std. 1484.11.2-2003 - ECMAScript API for content to runtime services communication

En este estándar (IEEE, 2003b) se describe una interfaz de programación de aplicaciones (API, del inglés Application Programming Interface) para el lenguaje ECMAScript para la comunicación entre el contenido de aprendizaje y los sistemas de ejecución. El objetivo de este estándar es lograr consenso, resolver ambigüedades y corregir defectos en las especificaciones actuales de API de ECMAScript para el intercambio de datos entre los contenidos de aprendizaje y los LMS.

IEEE std. 1484.11.3-2005 - XML Schema binding for content object communication

Este estándar (IEEE, 2005) define un esquema XML (eXtensible Markup Language) que permite representar el modelo de datos para la comunicación de objetos de contenido (LOM) definido en el estándar 1484.12.1.

\section{IEEE std. 1484.12.1-2002 - Learning object metadata}

Este estándar (IEEE, 2002) es la primera parte de un estándar IEEE dividido en varias partes que define metadatos para objetos de aprendizaje (LOM). En el estándar, una instancia de LOM describe características relevantes del objeto de aprendizaje al que se aplica, agrupadas en varias categorías: general, ciclo de vida, meta-metadatos, educativas, técnicas, derechos, relaciones, anotaciones y clasificación. 


\section{IEEE std. 1484.12.3-2005 - XML Schema for learning object metadata}

Este estándar (IEEE, 2005b) define un esquema XML para representar el modelo LOM definido en IEEE Std. 1484.12.1. El objetivo de este estándar es permitir crear instancias LOM en XML para facilitar su intercambio entre diferentes LMS.

\section{IEEE std. 1484.20.1-2007 - Reusable competency definitions}

Este estándar (IEEE, 2007b) se define un modelo de datos para describir, referenciar y compartir definiciones de competencias, fundamentalmente en el contexto de e-Learning. Se proporciona una representación formal de las características clave de una competencia, independientemente de su uso en un contexto particular. Permite la interoperabilidad entre sistemas de e-Learning que gestionen información sobre competencias, por ello guarda cierta relación con los trabajos de CETIS LEAP2A vistos anteriormente.

\section{IMS AccLIP - Accessibility for LIP}

Este es el primero, de una serie de documentos publicados por IMS GLC. Esta especificación técnica (IMS, 2003) define dos nuevos sub-esquemas para el paquete de información del aprendiz (IMS LIP, de Learner Information Package), que es otro documento de IMS GLC. Estos dos sub-esquemas proporcionan un modo de especificar preferencias de accesibilidad y acomodaciones (adaptaciones) para los usuarios. Las preferencias van más allá de dar soporte a las personas con discapacidad e incluyen otras necesidades de accesibilidad como la computación móvil, los entornos ruidosos, etc.

\section{IMS AccMD - Access for all meta-data specification}

Esta especificación de metadatos de acceso para todos (IMS, 2004) pretende facilitar la búsqueda de los recursos educativos que encajan con una declaración de preferencias o necesidades de usuario (documentadas mediante AccLIP).

Las necesidades y preferencias contempladas incluyen presentaciones alternativas de recursos, métodos alternativos para controlar recursos, recursos alternativos para recursos ya existentes y mejoras requeridas por el usuario. 
Esta especificación proporciona un lenguaje común para identificar y describir los recursos principales y sus alternativas para distintas modalidades de interacción y aprendizaje.

\section{IMS Digital Repositories}

Se trata de una especificación (IMS 2003b) definida mediante tres documentos (modelo de información, modelo XMLy mejores prácticas). Su objetivo es proporcionar recomendaciones para la interoperabilidad de las funciones más comunes de los repositorios de recursos digitales. La idea es que estas recomendaciones puedan implementarse en los servicios para permitirles ofrecer interfaces comunes.

\section{IMS ePortfolio}

El objetivo de esta especificación (IMS 2005) es conseguir que los "ePortfolios" (ver CETIS LEAP2A e IEEE 1484.20.1) sean interoperables entre diferentes sistemas e instituciones. Por ello da soporte al avance del aprendizaje permanente (LLL), facilita la transición de ePortfolios de la escuela al trabajo, permite que los educadores y las instituciones hagan un mejor seguimiento de las competencias, mejora la experiencia de aprendizaje y el desarrollo de los trabajadores.

\section{IMS GDALA - Guidelines for accessible learning applications}

Se trata de un informe (o white paper) que proporciona pautas para el desarrollo de aplicaciones de e-Learning accesibles (IMS 2002). Con este documento se proporciona un marco de trabajo para la comunidad de aprendizaje distribuido. Este marco de trabajo define qué soluciones existen, qué oportunidades y posibilidades hay para implementarlas, y qué áreas de trabajo de las tecnologías educativas necesitan más desarrollo e innovación para garantizar una educación que sea accesible para todos en cualquier momento y en cualquier lugar.

\section{IMS Learning Design}

La especificación de IMS LD (IMS 2003c) proporciona un lenguaje genérico y flexible para diseñar cursos usando cualquier tipo de enfoque pedagógico para e-Learning. Este lenguaje se ha diseñado para poder representar muchos métodos pedagógicos diferentes y con ello permite que sólo sea necesario implementar un único conjunto de herramientas de diseño y ejecución de contenidos educativos. 


\section{IMS Question \& Test Interoperability Specification}

La especificación QTI proporciona un lenguaje XML para representar preguntas y cuestionarios, de forma que permita la interoperabilidad del contenido con los sistemas de evaluación. La versión oficial más actualizada es la 2.0 del año 2005 (IMS, 2005b).

\section{ISO 9241-11O - Dialogue principles}

Este estándar internacional (ISO, 2006) está relacionado con la usabilidad y establece principios de diseño ergonómico formulados de forma general. También proporciona un marco de trabajo para aplicar estos principios al análisis, diseño y evaluación de sistemas interactivos. Los principios son: adecuación a la tarea, auto-descripción, conformidad con las expectativas del usuario, adecuación para el aprendizaje, control, tolerancia de errores y adecuación para la individualización.

\section{ISO 9241-129 - Individualization}

Este estándar en desarrollo (ISO, 2009) contendrá requisitos y recomendaciones sobre la ergonomía de la individualización de las interacciones persona-ordenador basadas en software. Existe una gran variedad de mecanismos básicos de individualización, cada uno de los cuales puede tener efectos positivos o negativos para los usuarios.

La individualización puede ser iniciada por el usuario (preferencias) o bien iniciada por el sistema (adaptación). El resultado de la individualización es una serie de cambios en la interfaz de usuario y en la interacción.

Este estándar incluirá pautas sobre: determinar cuándo es adecuado incluir individualización, seleccionar mecanismos adecuados de individualización, usar los distintos tipos de mecanismos de individualización y combinar esos tipos de mecanismos de individualización. 


\section{ISO 9241-151 - Web user interfaces}

Este estándar internacional (ISO, 2008) proporciona recomendaciones y pautas para el diseño centrado en el usuario de interfaces de usuario web, con el fin de incrementar su usabilidad. El estándar está centrado en cuatro aspectos del diseño de interfaces de usuario web: decisiones de alto nivel y estrategia de diseño, diseño de contenidos, navegación y búsqueda, presentación de contenido.

\section{ISO 9241-171 - Software accessibility}

Este estándar internacional (ISO, 2008b) proporciona requisitos y recomendaciones para el diseño de software accesible. Se aplica a la accesibilidad de sistemas interactivos y se dirige a muchos tipos de software (ofimática, web, sistemas de enseñanza, sistemas de bibliotecas, etc.).

Este documento promueve una usabilidad incrementada para un amplio rango de usuarios. Aunque el estándar no cubre el comportamiento o los requisitos de las tecnologías de apoyo (incluyendo software de apoyo), sí que aborda el uso de tecnologías de apoyo como una parte integrante de los sistemas interactivos.

Este estándar ha sido traducido al español y adoptado por AENOR como Norma Española, con el identificador UNE 139802 (AENOR, 2009).

\section{ISO 9241-2O Accessibility guidelines for ICT}

Este estándar (ISO, 2008c) proporciona recomendaciones generales para mejorar la accesibilidad de equipos y productos TIC. Este documento está diseñado para servir de punto de partida, para poder definir los requisitos específicos de cada tecnología cuando se diseñen productos accesibles. Si existe un estándar específico para un equipo o servicio (como el caso del software con el estándar ISO 9241-171), entonces los usuarios de esta norma internacional también pueden referirse a ese estándar más específico. 


\section{ISO/IEC 13066-1 Interoperability with assistive technology}

Este estándar en desarrollo (ISO/IEC, 2010) define las responsabilidades de las tecnologías de la información (IT) y de las unidades funcionales de las tecnologías de apoyo o ayudas técnicas (AT) para su interoperabilidad. Está centrado en la utilización de interfaces estándares y públicas para que el software de aplicación y los sistemas operativos puedan proporcionar información accesible a las AT.

ISO TR 22411 Ergonomics data and guidelines to address the needs of older persons and persons with disabilities

Este informe técnico (ISO, 2008d) presenta datos ergonómicos y pautas para aplicar la guía ISO/IEC 71 (ISO/IEC, 2001) al abordar las necesidades de las personas mayores y las personas con discapacidad en el desarrollo de nuevos estándares. Este informe proporciona datos ergonómicos y conocimiento sobre habilidades humanas (sensoriales, físicas y cognitivas) y alergias. También se incluyen pautas para el diseño accesible de productos, servicios y entornos.

\section{ISO/IEC24751-1 Framework for adaptability and accessibility in e-Learning}

Esta es la primera parte de un estándar dividido en tres. En la primera parte de ISO/IEC 24751 (ISO/IEC, 2008) se proporciona un marco de trabajo común para describir y especificar, por un lado, las necesidades y preferencias de los estudiantes y, por otro lado, la descripción correspondiente de los recursos educativos digitales. De esta forma las preferencias y necesidades de un individuo pueden equipararse con las herramientas de interfaz de usuario y los recursos digitales de aprendizaje más adecuados. El estándar 24751 (en sus tres partes) deriva de las especificaciones IMS AccLIP e IMS AccMD descritas anteriormente.

\section{ISO/IEC 24751-2 Access for all personal needs and preferences}

Esta segunda parte de ISO/IEC 24751 (ISO/IEC, 2008b) proporciona un modelo común de información para describir las necesidades y preferencias de los estudiantes o usuarios cuando acceden recursos o servicios servidos de forma digital. Esta descripción es una de las dos partes de las descripciones necesarias para equiparar las preferencias y necesidades de los usuarios con la entrega de contenidos educativos (tal y como se describe en ISO/IEC 24751-1). 


\section{ISO/IEC 24751-3 Access for all digital resource description}

Esta tercera parte de ISO/IEC 24751 (ISO/IEC, 2008c) proporciona un lenguaje común para describir recursos digitales de aprendizaje de forma que se facilite la equiparación de esos recursos con las necesidades y preferencias de los estudiantes (tal y como se definen en ISO/IEC 24751-2). Esta descripción es la segunda de las dos descripciones necesarias para equiparar las preferencias y necesidades de los usuarios con la entrega de contenidos educativos (tal y cómo se describe en ISO/IEC 24751-1).

\section{ISO/IEC 24752-1 Framework of the universal remote console}

Ésta es la primera parte de un estándar dividido en 5 partes. El estándar en su conjunto facilita el manejo de productos de información y electrónicos a través de interfaces remotas y alternativas y con agentes inteligentes. En este estándar los dispositivos y servicios que son controladas se denominan "objetivos" ("targets") y los dispositivos de control y sus interfaces se denominan "consolas remotas universales" ("universal remote consoles").

En esta primera parte de ISO/IEC 24752 (ISO/IEC, 2008d) se define un marco de trabajo de componentes que se combinan para facilitar interfaces de usuario remotas y el control remoto de dispositivos electrónicos y servicios accesibles por red a través de una consola remota universal (URC).

\section{ISO/IEC 24752-2 URC user interface socket description}

Esta segunda parte de ISO/IEC 24752 (ISO/IEC, 2008e) describe los “sockets" de interfaz de usuario, un concepto abstracto que describe la funcionalidad y el estado de un dispositivo o servicio (objetivo - target) de forma que pueda ser interpretado por una máquina. También define un lenguaje basado en XML para describir un "socket" de interfaz de usuario.

\section{ISO/IEC 24752-3 URC presentation template}

Esta tercera parte de ISO/IEC 24752 (ISO/IEC, 2008f) define un lenguaje (lenguaje de marcado para plantillas de presentación - presentation template markup language) para describir plantillas de presentación; es decir, especificaciones de interfaces de usuario que sean independientes de la modalidad de interacción con 
el usuario. Estas plantillas de presentación están asociadas con descripciones de "sockets" de interfaz de usuario (ISO/IEC 24752-2).

\section{ISO/IEC 24752-4 URC target description}

Esta cuarta parte de ISO/IEC 24752 (ISO/IEC, 2008g) define un lenguaje basado en XML para la descripción de los objetivos (targets) y sus "sockets", tal y como se usan en el marco de trabajo de URC para facilitar el descubrimiento de las funciones y servicios de los dispositivos. Una descripción de objetivo (target description) es un documento realizado en este lenguaje.

\section{ISO/IEC 24752-5 URC resource description}

Esta quinta parte de ISO/IEC 24752 (ISO/IEC, 2008h) define una sintaxis para describir recursos atómicos, hojas de recursos, descripciones de implementación de interfaces de usuario, servicios de recursos y directorios de recursos, todos ellos relevantes para la interfaz de usuario de un dispositivo o servicio (objetivo o target).

\section{ISO/IEC 24756 Common access profile}

Este estándar internacional (ISO/IEC, 2009b) define un marco de trabajo para especificar un perfil común de acceso (CAP, del inglés Common Access Profile) de las necesidades y capacidades de los usuarios, sistemas de computación y sus entornos, incluyendo el acceso proporcionado por las tecnologías de apoyo. Proporciona una base para identificar y gestionar de forma estandarizada los problemas de accesibilidad encontrados en distintas plataformas. Puede utilizarse para evaluar la accesibilidad para usuarios concretos de sistemas existentes en entornos determinados.

\section{ISO/IEC 24786 Accessible user interface for accessibility settings}

Este estándar internacional (ISO/IEC, 2009c) especifica requisitos y recomendaciones para lograr que la configuración de accesibilidad sea, a su vez, accesible. Proporciona orientación sobre determinados parámetros de accesibilidad, sobre cómo acceder y operar en el modo de configuración de accesibilidad y sobre cómo activar de forma rápida algunas características de accesibilidad. Este estándar se aplica fundamentalmente a los sistemas operativos. 


\section{ISO/IEC TR 29138-1 Accessibility: user needs summary}

Ésta es la primera parte de un informe técnico dividido en tres partes. En la primera parte (ISO/IEC, 2009d) se identifica una colección de necesidades de usuario de las personas con discapacidad, con el fin de que los desarrolladores de estándares tengan en cuenta esas necesidades para desarrollar o revisar sus estándares. Estas necesidades de usuario también son útiles para los desarrolladores de productos y servicios de tecnologías de la información, así como para las personas que defienden la necesidad de tener productos y servicios accesibles.

\section{ISO/IEC TR 29138-2 Accessibility: standards inventory}

En esta segunda parte (ISO/IEC, 2009) se identifica una colección de documentos (llamados estándares, aunque se incluyen documentos que no son estándares tradicionales de ISO o de ISO/IEC) que proporcionan pautas para cubrir las necesidades de las personas con discapacidad.

\section{ISO/IEC TR 29138-3 Accessibility: user needs mapping}

En esta tercera parte (ISO/IEC, 2009e) se proporciona una guía para equiparar las necesidades de los usuarios con las cláusulas de un determinado estándar, informe técnico o conjunto de pautas.

\section{$W_{3} C C C / P P$ - Composite capability / preference profiles}

Esta recomendación del Consorcio de la Web $\left(\mathrm{W}_{3} \mathrm{C}, 2004\right)$ define un formato de datos para representar perfiles de cliente y un marco de trabajo para incorporar características específicas de las aplicaciones y de los entornos operativos. Un perfil CC/PP (composición de capacidades/perfiles de preferencias) es una descripción de las capacidades de un dispositivo y de las preferencias de sus usuarios. Normalmente se denomina contexto de entrega del dispositivo y se puede utilizar para dirigir la adaptación de contenido que se presenta en ese dispositivo. Se utiliza el lenguaje RDF (Resource Description Framework) para crear los perfiles que describen las capacidades de las aplicaciones de usuario (navegadores web, entre otros) y las preferencias de sus usuarios.

\section{W3C ATAG - Authoring tool accessibility}

Esta recomendación del $\mathrm{W}_{3} \mathrm{C}\left(\mathrm{W}_{3} \mathrm{C}, 2000\right)$ proporciona pautas de accesibilidad para los desarrolladores de herramientas de autor: los programas que se utilizan para 


\section{R. Fabregat; G. Moreno; F. Amo; J. Fuertes; A. GonzÁlez; L. Martínez}

ESTÁNDARES PARA E-LEARNING ADAPTATIVO Y ACCESIBLE

crear contenidos en la web. Estas pautas tienen dos objetivos: en primer lugar, se trata de conseguir que las herramientas de autor ayuden a crear contenido accesible; en segundo lugar se trata de conseguir que esas herramientas de autor tengan una interfaz de usuario accesible.

\section{$W_{3} C U A A G-U s e r$ agent accessibility}

Esta recomendación del $\mathrm{W}_{3} \mathrm{C}\left(\mathrm{W}_{3} \mathrm{C}, 2002\right)$ proporciona pautas de accesibilidad para los desarrolladores de agentes de usuario (las aplicaciones que se utilizan para acceder a los contenidos web, como los navegadores o los reproductores multimedia). Un agente de usuario que cumpla con estas pautas promoverá la accesibilidad a través de su propia interfaz de usuario, así como por otros medios, como la habilidad para comunicarse con las tecnologías de apoyo. Finalmente, todos los usuarios, y no sólo los usuarios con discapacidad, deberían percibir que los agentes de usuario que cumplen con estas pautas son más usables.

\section{W3C WCAG - Web content accessibility}

Esta recomendación del $\mathrm{W}_{3} \mathrm{C}\left(\mathrm{W}_{3} \mathrm{C}, 2008\right)$ proporciona pautas de accesibilidad para conseguir que el contenido web sea más accesible. Si se siguen estas pautas se conseguirá que el contenido sea accesible para un rango más amplio de personas con discapacidad. Las pautas están definidas de forma que son, al mismo tiempo, verificables e independientes de las tecnologías web. En documentos adicionales a las WCAG 2.0 se proporcionan guías para cumplir con los criterios de conformidad en varias tecnologías web.

\section{Análisis de los estándares}

Una vez que los estándares fueron seleccionados de acuerdo a los criterios anteriores, se decidió clasificarlos para obtener una imagen más clara de su alcance. Los criterios de clasificación en este caso fueron dos:

- De acuerdo con la orientación hacia el usuario (Martínez, 2007), los estándares pueden ser:

o Centrados en el usuario (U), ofrecen orientación sobre la accesibilidad, el diseño para todos y la usabilidad general, desde el punto de vista de los usuarios del producto. 
o Centrados en los desarrolladores (D), son de carácter más técnico y ofrecen soluciones técnicas que los desarrolladores pueden utilizar para crear productos.

- Dependiendo de las áreas de modelado con las que están relacionados:

o Modelado de Contenido (C)

o Modelado de Usuarios (U)

o Modelado de Dispositivos (D), incluyendo hardware y software

- Modelado de Adaptaciones (A)

o Modelado de la Interfaz de Usuario (IU)

En la tabla 1 se recoge la clasificación de los estándares en función de los dos criterios expuestos. $\mathrm{Y}$ en la tabla 2 se muestra un resumen cuantitativo de los resultados obtenidos con este análisis.

Según los resultados podemos decir que:

- Hay una tendencia clara de los estándares analizados por orientar sus esfuerzos bien hacia los usuarios, bien hacia los desarrolladores, pero rara vez a ambos simultáneamente.

\begin{tabular}{|c|c|c|c|c|c|c|c|}
\hline \multirow[t]{2}{*}{ Estándar } & \multicolumn{2}{|c|}{ Orientación } & \multicolumn{5}{|c|}{ Áreas de modelado en A2UN@ } \\
\hline & U & D & C & U & D & A & IU \\
\hline ADL SCORM & & $x$ & $x$ & & & & $x$ \\
\hline CETIS LEAP2A & & $\mathrm{x}$ & & $x$ & & & \\
\hline ETSI EG 202116 V1.2.1 & $x$ & & & & $x$ & & $x$ \\
\hline ETSI ES 202746 & & $x$ & & $x$ & & $x$ & \\
\hline IEEE std. 1484.1-2003 & & $x$ & & & & & \\
\hline IEEE std. 1484.4-2007 & & $x$ & $x$ & & & & \\
\hline IEEE std. 1484.11.1-2004 & & $x$ & $x$ & & & $x$ & \\
\hline IEEE std. 1484.11.2-2003 & & $x$ & $x$ & & & $x$ & \\
\hline IEEE std. 1484.11.3-2003 & & $x$ & $x$ & & & $x$ & \\
\hline IEEE std. 1484.12.1-2002 & & $\mathrm{x}$ & $x$ & & & & \\
\hline IEEE std. 1484.12.3-2005 & & $x$ & $x$ & & & & \\
\hline IEEE std. 1484.20.1-2007 & & $x$ & & $x$ & & $x$ & \\
\hline
\end{tabular}


R. Fabregat; G. Moreno; F. Amo; J. Fuertes; A. GonzÁLez; L. Martínez ESTÁNDARES PARA E-LEARNING ADAPTATIVO Y ACCESIBLE

\begin{tabular}{|c|c|c|c|c|c|c|c|}
\hline IMS AccLIP & & $x$ & & \multicolumn{2}{|l|}{$x$} & \multicolumn{2}{|l|}{$x$} \\
\hline IMS AccMD & & $x$ & $x$ & & & $x$ & \\
\hline IMS Digital Repositories & & $x$ & $x$ & & & $\mathrm{x}$ & \\
\hline IMS ePortfolio & & $x$ & & $x$ & & $\mathrm{x}$ & \\
\hline IMS GDALA & $x$ & & & & & & \\
\hline IMS LD & & $x$ & $\mathrm{x}$ & & & & \\
\hline IMS QTI & & $x$ & $x$ & & & & \\
\hline ISO $9241-110$ & $x$ & & & & & & $\mathrm{x}$ \\
\hline ISO 9241-129 & $x$ & & & $x$ & & $\mathrm{x}$ & \\
\hline ISO 9241-151 & $x$ & & $\mathrm{x}$ & & & & \\
\hline ISO 9241-171 & $x$ & & $\mathrm{x}$ & & $x$ & & $x$ \\
\hline ISO 9241-20 & $x$ & & & & $\mathrm{x}$ & & $\mathrm{x}$ \\
\hline ISO/IEC 13066-1 & & $x$ & & & & & $\mathrm{x}$ \\
\hline ISO TR 22411 & $x$ & & & & & & $\mathrm{x}$ \\
\hline ISO/IEC 24751 & & $x$ & $\mathrm{x}$ & $x$ & $\mathrm{x}$ & & \\
\hline ISO/IEC 24752 & & $x$ & & & $x$ & & $\mathrm{x}$ \\
\hline ISO/IEC 24756 & & $x$ & $x$ & $x$ & $\mathrm{x}$ & $\mathrm{x}$ & $\mathrm{x}$ \\
\hline ISO/IEC 24786 & $x$ & $x$ & & & & $x$ & $\mathrm{x}$ \\
\hline ISO/IEC TR 29138 & $x$ & & & & & $x$ & $x$ \\
\hline W3C CC/PP & & $x$ & & $x$ & $x$ & & \\
\hline W3C WAI ATAG & $x$ & $x$ & $\mathrm{x}$ & & $x$ & & $\mathrm{x}$ \\
\hline W3C WAI UAAG & $x$ & $x$ & & & $x$ & & $\mathrm{x}$ \\
\hline W3C WAI WCAG 2.0 & $x$ & & $\mathrm{x}$ & & & $\mathrm{x}$ & \\
\hline
\end{tabular}

Tabla 1. Clasificación de los estándares paraA2UN@

- Dentro de las áreas de modelado hay una clara tendencia por abordar el modelado del contenido, de la adaptación y de la interfaz de usuario. El modelado de usuario y de los dispositivos está mucho menos representado. 


\begin{tabular}{cccc}
\hline Totales & $\begin{array}{c}\text { Estándares centrados } \\
\text { en el usuario }\end{array}$ & $\begin{array}{c}\text { Estándares centrados } \\
\text { en el desarrollador }\end{array}$ & $\begin{array}{c}\text { Estándares centrados } \\
\text { en usuario y } \\
\text { desarrollador }\end{array}$ \\
\hline $\mathbf{C}=\mathbf{1 7}$ & $\mathbf{I U}=\mathbf{7}$ & $\mathbf{C}=\mathbf{1 3}$ & $\mathbf{I U}=\mathbf{3}$ \\
$\mathbf{A}=\mathbf{1 5}$ & $\mathrm{D}=3$ & $\mathbf{A}=\mathbf{1 1}$ & $\mathbf{D}=\mathbf{2}$ \\
$\mathbf{I U}=\mathbf{1 4}$ & $\mathrm{C}=3$ & $\mathrm{U}=8$ & $\mathrm{~A}=1$ \\
$\mathrm{U}=9$ & $\mathrm{~A}=3$ & $\mathbf{I U}=\mathbf{4}$ & $\mathbf{C}=\mathbf{1}$ \\
$\mathrm{D}=8$ & $\mathbf{U}=\mathbf{0}$ & $\mathbf{D}=\mathbf{3}$ & $\mathbf{U}=\mathbf{0}$ \\
\hline
\end{tabular}

Tabla 2. Resultados de la clasificación por áreas de modelado

- Los estándares actuales de usabilidad y la accesibilidad centrados sólo en el usuario tienen una fuerte orientación hacia el modelo de interfaz de usuario. Esto contrasta con su falta de apoyo al modelado del usuario. La principal razón de esto es que ellos proveen soluciones desde la perspectiva de los usuarios y no soluciones técnicas para los desarrolladores.

- Los estándares actuales de usabilidad y accesibilidad centrados sólo en el desarrollador tienen una fuerte orientación hacia los modelos de contenido y de adaptación. Esto contrasta con su falta de soporte del modelado de interfaces de usuario y de dispositivos.

- Los estándares que están centrados tanto en el usuario como en el desarrollador están más orientados al modelado de la interfaz de usuario y de los dispositivos.

\section{CONCLUSIONES Y TRABAJO FUTURO}

Después del análisis de estándares existentes, se puede concluir que hay una falta de estándares que estén orientados al mismo tiempo hacia los usuarios y los desarrolladores y que tengan en cuenta todas las áreas de modelado. Dentro de estas áreas de modelado, las dos que tienen menor presencia son el modelado del usuario y el modelado de los dispositivos, dos áreas que son esenciales dentro del paradigma del aprendizaje permanente. Por lo tanto, desde el proyecto A2UN@ se trabajará en el desarrollo de estándares internacionales que cubran las carencias detectadas.

Por otro lado, desde el proyecto se pretende desarrollar un modelo conceptual de sistemas de aprendizaje permanente que esté basado en estándares, que cubra las dos orientaciones (usuarios y desarrolladores) y los cinco tipos de modelado. El objetivo de dicho modelo conceptual consistirá en facilitar la interoperabilidad entre los estándares que representan visiones parciales de un sistema de aprendizaje permanente. 
La figura 1 muestra una representación esquemática del resultado que se pretende obtener con dicho modelo. Este modelo debe representar todos los elementos básicos de un sistema de aprendizaje permanente, las relaciones entre todos esos elementos y, finalmente, una equiparación entre esos elementos y los requisitos y recomendaciones de los estándares relevantes.

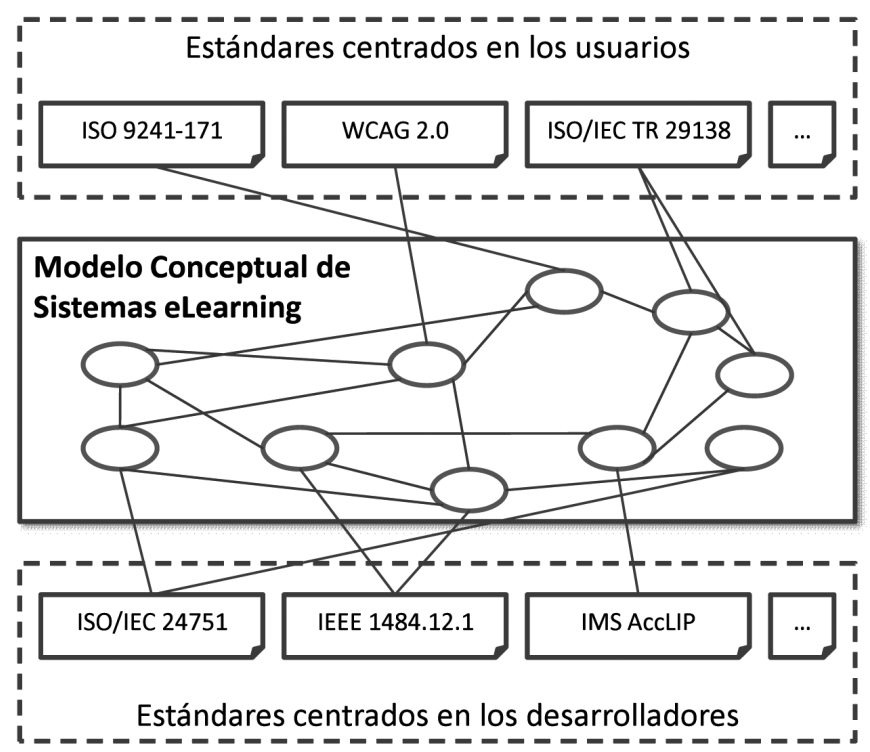

Figura 1. Modelo conceptual de sistemas de aprendizaje permanente

Esta tarea es muy compleja por dos razones fundamentales. En primer lugar, existen varios estándares que podrían aplicarse a cada elemento o conjunto de elementos de un sistema de aprendizaje permanente y muchas veces estos estándares entran en conflicto unos con otros. Por ejemplo, en el área de modelado de dispositivos hay al menos ocho estándares diferentes que se podrían aplicar, pero que tienen diferentes enfoques.

En segundo lugar, hay estándares que se pueden aplicar a varias áreas de trabajo. Por ejemplo, el estándar internacional 9241-20 que tiene orientación de usuarios se puede aplicar, tanto al modelado de dispositivos, como al modelado de interfaces de usuario.

En cualquier caso, se aprecia una necesidad clara: el modelo debe proporcionar un vocabulario común y genérico para describir sistemas de aprendizaje permanente 
y sus componentes. Y ese vocabulario debe diseñarse para facilitar al máximo la equiparación entre el modelo y los estándares relevantes.

\section{Agradecimientos}

Este trabajo está parcialmente financiado por el proyecto A2UN@ (TIN20o8o6862-C04-01/TSI, TIN2008-06862-Co4-02/TSI).

\section{REFERENCIAS BIBLIOGRÁFICAS}

ADL (2009) SCORM 2004 4th Edition Version 1.1 (4th Ed.). [en línea] Disponible en: http://www.adlnet.gov/ Technologies/scorm/SCORMSDocuments/2004\%204th\%20Edition/Documentation.aspx (consulta 2010, 10de abril).

AENOR(2009).UNE139802:2009. Requisitos de Accesibilidad de Software. Norma Española (adopción de EN ISO 9241-171).

CETIS (2009) The LEAP2A specification for portability and interoperability of $e-$ portfolio information. 2009-03 version. [en línea] Disponible en: http://wiki. cetis.ac.uk/2009-03/Leap2A specification) (consulta 2010, 10de abril).

ETSI (2002) ETSI EG 202116 V1.2.1: Human Factors (HF); Guidelines for ICT products and services; "Design for All". ETSI Guide.

ETSI (2010) ETSI ES 202746 V1.1.1: Human Factors (HF); Personalization and User Profile Management; User Profile Preferences and Information. ETSI Standard.

Hodgkinson, R. (2009) 9th Report on International ICT Accessibility Standards Proposed, Being Developed and Recently Published [en línea] Disponible en: http://www.tiresias. org/research/standards/report 10.htm (consulta 2010, 10de abril).

IEEE (2002) IEEE Std. 1484.12.1-2002. IEEE
Standard for Learning Object Metadata. IEEE (2003). IEEE std. 1484.1-2003. IEEE Standard for Learning TechnologyLearning Technology Systems Architecture (LTSA).

IEEE (2003b). IEEE std. 1484.11.2-2003. Standard for Learning Technology-ECMAScript Application Programming Interface for Content to Runtime Services Communication.

IEEE (2004). IEEE std. 1484.11.1-2004. IEEE Standard for Learning TechnologyData Model for Content to Learning Management System Communication.

IEEE (2005). IEEE std. 1484.11.3-2005. IEEE Standard for Learning TechnologyExtensible Markup Language (XML) Schema Binding for Data Model for Content Object Communication.

IEEE (2005b). IEEE std. 1484.12.3-2005. IEEE Standard for Learning TechnologyExtensible Markup Language (XML) Schema Definition Language Binding for Learning Object Metadata.

IEEE (2007). IEEE std. 1484.4-2007. TrialUse Recommended Practice for Digital Rights Expression Languages (DRELs) Suitable for eLearning Technologies. IEEE Recommended Practice.

IMS Global Learning Consortium (2002). IMS Guidelines for Developing Accessible Learning Applications. Version 1. White Paper.

IMS Global Learning Consortium (2003). 
IMS Learner Information Package Accessibility for LIP. Version 1. Final Specification.

IMS Global Learning Consortium (2003b). IMS Digital Repositories Specification. Version 1. Final Specification.

IMS Global Learning Consortium (2003c). IMS Learning Design. Version 1. Final Specification.

IMS Global Learning Consortium (2004). IMS AccessForAll Meta-data Information Model Specification. Version 1. Final Specification.

IMS Global Learning Consortium (2005). IMS ePortfolio. Version 1. Final Specification.

IMS Global Learning Consortium (2005b). IMS Question and Test Interoperability . Version 2. Final Specification.

ISO (2006). ISO 9241-11O Ergonomics of human-system interaction -- Part 110: Dialogue principles. ISO Standard.

ISO (2008). ISO 9241-151 Ergonomics of human-system interaction -- Part 151: Guidance on World Wide Web user interfaces. ISO Standard.

ISO (2008b). ISO 9241-171 Ergonomics of human-system interaction -- Part 171: Guidance on software accessibility. ISO Standard.

ISO (2008c). ISO 9241-2O Ergonomics of human-system interaction -- Part 20: Accessibility guidelines for information/ communication technology (ICT) equipment and services. ISO Standard.

ISO (2008d). ISO TR 22411 Ergonomics data and guidelines for the application of ISO/IEC Guide 71 to products and services to address the needs of older persons and persons with disabilities. ISO Technical Report.

ISO (2009). ISO DIS 9241-129 Ergonomics of human-system interaction -- Part 129: Guidance on software individualization. ISO Draft International Standard.

ISO/IEC (2001). ISO/IEC Guide 71 Guidelines for standards developers to address the needs of older persons and persons with disabilities. ISO/IEC Guide.
ISO/IEC (2008). ISO/IEC 24751-1. Information technology--Individualized adaptability and accessibility in e-learning, education and training -Part 1: Framework and reference model. ISO/IEC Standard.

ISO/IEC (2008b). ISO/IEC 24751-2. Information technology--Individualized adaptability and accessibility in e-learning, education and training -Part 2: “Access for all” personal needs and preferences for digital delivery. ISO/ IEC Standard.

ISO/IEC (2008c). ISO/IEC 24751-3. Information technology -- Individualized adaptability and accessibility in e-learning, education and training -- Part 3: "Access for all" digital resource description. ISO/IEC Standard.

ISO/IEC (2008d). ISO/IEC 247521. Information technology -- User interfaces -- Universal remote console -- Part 1: Framework. ISO/IEC Standard.

ISO/IEC (2008). ISO/IEC 24752-2. Information technology -- User interfaces -- Universal remote console -Part 2: User interface socket description. ISO/IEC Standard.

ISO/IEC (2008f). ISO/IEC 24752-3. Information technology -- User interfaces -- Universal remote console -Part 3: Presentation template. ISO/IEC Standard.

ISO/IEC (2008g). ISO/IEC 24752-4. Information technology -- User interfaces -- Universal remote console -- Part 4: Target description. ISO/IEC Standard.

ISO/IEC (2008h). ISO/IEC 247525. Information technology -- User interfaces -- Universal remote console -- Part 5: Resource description. ISO/IEC Standard.

ISO/IEC (2009). ISO/IEC TR 29138-2. Information technology -- Accessibility considerations for people with disabilities -- Part 2: Standards inventory. ISO/IEC Technical Report.

ISO/IEC (2009b). ISO/IEC 24756. Framework for specifying a Common 
Access Profile (CAP) of needs and capabilities of users, systems and their environments. ISO/IEC Standard.

ISO/IEC (2009c). ISO/IEC 24786. Information technology -- User interfaces -- Accessible user interface for accessibility settings. ISO/IEC Standard.

ISO/IEC (2009d). ISO/IEC TR 29138-1. Information technology -- Accessibility considerations for people with disabilities - Part 1: User Needs Summary. ISO/IEC Technical Report.

ISO/IEC (2009e). ISO/IEC TR 29138-3. Information technology -- Accessibility considerations for people with disabilities - Part 3: Guidance on user needs mapping. ISO/IEC Technical Report.

ISO/IEC (2010). ISO/IEC FCD 13066 Information Technology - Interoperability with Assistive Technology (AT) -- Part 1: Requirements and recommendations for interoperability. ISO/IEC Final Committee Draft.

Martínez Normand, Loïc (2007). Software
Accessibility Standards, User Modelling and Adaptive Systems. TUMAS- $A$ Workshop's Proceedings. 11-16.

$\mathrm{W}_{3} \mathrm{C}$ (2000). Authoring Tool Accessibility Guidelines 1.o. World Wide Web Consortium Recommendation. [en línea] Disponible en: http://www.w3.org/TR/ ATAG10/ (consulta 2010, 10de abril)

$\mathrm{W}_{3} \mathrm{C}$ (2002). User Agent Accessibility Guidelines 1.o. World Wide Web Consortium Recommendation. [en línea] Disponible en: http://www.w3.org/TR/ WAI-USERAGENT/ (consulta 2010, 1ode abril)

W3C (2004). Composite Capability/ Preference Profiles (CC/PP): Structure and Vocabularies 1.o. World Wide Web Consortium Recommendation. [en línea] Disponible en: http://www.w3.org/TR/ CCPP-struct-vocab/ (consulta 2010, 1ode abril).

$\mathrm{W}_{3} \mathrm{C}$ (2008). Web Content Accessibility Guidelines 2.o. World Wide Web Consortium Recommendation. [en línea] Disponible en: http://www.w3.org/TR/ WCAG/ (consulta 2010, 10de abril). 


\section{PERFIL ACADÉMICO Y PROFESIONAL DE LOS AUTORES}

Fernando Alonso Amo es Ingeniero Industrial, Licenciado en Informática y Doctor en Informática por la Universidad Politécnica de Madrid. Actualmente es Catedrático de Universidad en el área de Ciencias de la Computación e Inteligencia Artificial y responsable del Grupo de Investigación CETTICO. Asimismo, pertenece al Cuerpo Superior de Sistemas y Tecnologías de la Información de la Administración del Estado. Su actividad investigadora se centra en los modelos de desarrollo de software y la enseñanza b-elearning.

E-mail: falonso@fi.upm.es

Ramón Fabregat Gesa es Ingeniero en Informática y $\mathrm{PhD}$ en Ingenieria Industrial. Profesor del departamento de Arquitectura y Tecnología de Computadores e investigador del Instituto de Informática y Aplicaciones de la Universidad de Girona (UdG). Miembro del grupo de Comunicaciones y Sistemas Distribuidos (BCDS) y responsable del laboratorio de Sistemas Hipermedia Adaptativos. Actualmente es investigador principal por la UdG en el proyecto “A2UN@: Accessibility and Adaptation for ALL in Higher Education”.

E-mail: $\underline{\text { rfabregas@fi.upm.es }}$

José Luis Fuertes Castro es Doctor en Informática por la Universidad Politécnica de Madrid y Profesor en la Facultad de Informática de dicha Universidad. Es director adjunto de la Sección de Transferencia Informática en Apoyo a las Minusvalías del Centro de Transferencia Tecnológica en Informática y Comunicaciones. Ha participado en más de 35 proyectos de investigación y es autor de más de 60 publicaciones. Coordina el CTN139/SC8/GT1 de AENOR (Interfaces de Usuario para Personas con Discapacidad.

E-mail: jfuertes@fi.upm.es

Ángel Lucas González Martínez es Licenciado en Informática y Doctor en Informática por la Universidad Politécnica de Madrid (UPM). Actualmente es Profesor Contratado, Doctor de la UPM. Sus campos principales de investigación son las aplicaciones en apoyo a la enseñanza a niños con necesidades educativas especiales y los sistemas de realidad virtual de escritorio para aplicaciones docentes, de ingeniería o comerciales.

E-mail: agonzalez@fi.upm.es

Loïc Martínez Normand es Licenciado en Informática y Doctor en Informática por la Universidad Politécnica de Madrid (UPM). Actualmente es Profesor Contratado Doctor de la UPM. Es coautor de cuatro libros, ocho capítulos 
de libro y más de 60 artículos publicados en revistas y congresos internacionales. Sus líneas de investigación son: accesibilidad de las TIC para personas con discapacidad, metodologías de desarrollo de software y técnicas de data mining. Ha participado en más de 40 proyectos nacionales o europeos.

E-mail: loic@fi.ump.es

Germán Darío Moreno García es Ingeniero de Sistemas por la Universidad Industrial de Santander, Colombia (2003) y Máster por la Universidad de Girona, España (2008). Está estudiando el Doctorado en Tecnologías de la Información en la Universidad de Girona. Sus intereses de investigación abarcan temas relacionados con la accesibilidad web y la adaptación en eLearning para personas con necesidades especiales de interacción. Actualmente es investigador por la UdG en el proyecto “A2UN@: Accessibility and Adaptation for ALL in Higher Education”.

E-mail: gmoreno@eia.udg.edu

DIRECCCIÓN DE LOS AUTORES

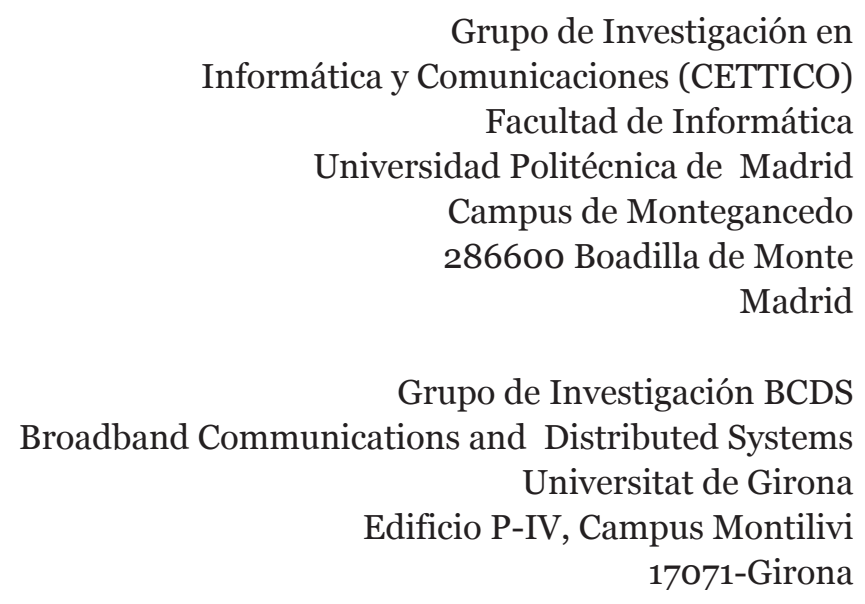

Fecha de recepción del artículo: 10/05/10

Fecha de aceptación del artículo: 17/07/10 